\title{
Improvement of quality of life in patients surgically treated for asymptomatic unruptured intracranial aneurysms
}

\author{
Shigeo Yamashiro, Toru Nishi, Kazunari Koga, Tomoaki Goto, Masatomo Kaji, Daisuke Muta, Jun- \\ ichi Kuratsu, Shodo Fujioka
}

J Neurol Neurosurg Psychiatry 2007;78:497-500. doi: 10.1136/jnnp.2006.098871

See end of article for authors' affiliations

Correspondence to:

S Yamashiro, Kumamoto

Rosai Hospital, 1670

Takehara-machi, Yatsushiro,

Kumamoto 866-8533,

Japan; KHC04160@

nifty.com

Received 5 June 2006

Revised 26 November 2006

Accepted 28November 2006

Published Online First

18 December 2006
Objective: To compare the preoperative and postoperative health-related quality of life (QOL) and psychological state of patients with asymptomatic unruptured intracranial aneurysms (ICAs) who underwent elective surgery.

Methods: Out of 67 patients who underwent neck clipping of ICAs, we assessed the QOL of 61 patients using Short Form-36 (SF-36); their psychological state was rated on the Hospital Anxiety and Depression Scale (HADS) before, 3 months, and 1 and 3 years after treatment.

Results: The preoperative mean scores for each of the eight SF-36 domains except bodily pain were significantly lower in the study population than in the reference population. $14(20.9 \%)$ patients experienced surgical complications defined as neurological deterioration and/or abnormal CT findings within 30 days of the operation. Despite some complications, the QOL of all operated patients returned to the mean level of the reference population 3 years after treatment. At 3 months after surgery, the scores for psychosocial activities and general health perception were transiently below the preoperative levels. According to the HADS, the patients experienced mild anxiety before the operation; it disappeared by the third postoperative month.

Conclusions: Preoperatively, patients with unruptured ICAs reported a significantly decreased QOL. It further declined transiently after elective surgery, but it returned to the mean level recorded for the reference population within 3 years. Our findings suggest that these patients derived significant $Q O L$ benefits from their surgery. Hence subjective QOL issues should be considered in deciding whether treatment-related risks and their natural history, such as their potential rupture, warrant surgery of asymptomatic unruptured ICAs.
$\mathrm{S}$ ome patients with unruptured intracranial aneurysms (ICAs) undergo elective microsurgical clipping to prevent subarachnoid haemorrhage (SAH) that may result in a poor prognosis. Bederson et al ${ }^{1}$ suggested that a history of SAH and the presence of aneurysms with diameter $>10 \mathrm{~mm}$ render patients with unruptured aneurysms, especially those with symptomatic lesions, eligible for surgical intervention. A systematic review of unruptured ICAs in Japan showed that the risk of rupture is significantly higher than that reported by international large-scale studies. ${ }^{2}$ Therefore, patients with asymptomatic and/or small $(<10 \mathrm{~mm})$ aneurysms tend to be treated surgically in Japan.

The international guidelines for the management of unruptured ICAs are based on their natural history and surgical results, ${ }^{3-5}$ and rates of surgical morbidity and mortality are considered objective epidemiological outcome measures. However, subjective assessments and patient-based outcomes have gained attention in the evaluation of functional outcomes after treatment. Judgement parameters include anxiety, satisfaction with treatment and health-related quality of life (QOL) issues. In neurosurgical outcome evaluations, the QOL after surgery for $\mathrm{SAH}^{6-8}$ vertebral artery dissection ${ }^{9}$ and acoustic neuroma ${ }^{10}$ has been assessed using universal health-related QOL measures. Many patients with unruptured ICAs undergo elective surgery because they experience serious anxiety regarding their possible rupture and a consequent QOL decline. Although from a patient's standpoint improved QOL is the goal of treatment, this issue has not been addressed adequately in the literature.

Our previous retrospective study of long-term health-related QOL, anxiety and depression in patients who had undergone elective aneurysm clipping at our institute during the past 10 years ${ }^{11}$ showed that most of the patients experienced a QOL similar to that of the general population. In the current prospective investigation, we assessed the preoperative and postoperative QOL of patients with unruptured ICAs who underwent elective surgery.

\section{METHODS}

Patient population and procedures

This prospective study was performed with the approval of the ethical committee at Saiseikai Kumamoto Hospital, Kumamoto Japan. Between April 2002 and April 2003, 108 patients with unruptured ICAs underwent surgery at our institute; 67 of these voluntarily agreed to participate in our pretreatment and post-treatment QOL survey. We excluded patients with symptomatic unruptured aneurysms and those who underwent treatment for ruptured and unruptured aneurysms during the same admission. The participating patients were surveyed at four time points - that is, 2 days before, and 3 months and 1 and 3 years after the operation. In patients who underwent more than one surgical procedure, follow-up was started after the last operation.

\section{Treatment}

After craniotomy, unruptured aneurysms located in the anterior circulation and the basilar bifurcation were approached via the conventional pterional transylvian or interhemispheric route. In aneurysms arising from the vertebral artery, we used a lateral suboccipital approach. Patients with multiple bilateral aneurysms underwent craniotomy twice after an interval of more than 3 months.

Abbreviations: HADS, Hospital Anxiety and Depression Scale; ICAs, intracranial aneurysms; mRS, modified Rankin Score; QOL, quality of life; SAH, subarachnoid haemorrhage; SF-36, Short Form-36 
Table 1 Operative complications and functional outcomes

\begin{tabular}{|c|c|c|}
\hline & \multicolumn{2}{|l|}{ CT findings } \\
\hline \multirow{4}{*}{$\begin{array}{l}\text { Perioperative complications } \\
\text { Seizure } \\
\text { Transient dysphasia } \\
\text { Mental disturbance† } \\
\text { Asymptomatic }\end{array}$} & Yes & No \\
\hline & 1 (ischaemia) & \\
\hline & 1 (ischaemia) & 2 \\
\hline & $\begin{array}{l}1 \text { (ischaemia) } \\
1 \text { (contusion) }\end{array}$ & \\
\hline \multirow{2}{*}{\multicolumn{2}{|c|}{$\begin{array}{l}\text { Visual loss in one eyef } \\
\text { Perioperative death of unknown } \\
\text { causes }\end{array}$}} & 1 \\
\hline & & 1 \\
\hline \multicolumn{3}{|l|}{ Other complications } \\
\hline \multirow{2}{*}{\multicolumn{2}{|c|}{$\begin{array}{l}\text { Meningitis } \\
\text { Chronic subdural hematoma }\end{array}$}} & 2 \\
\hline & & $3^{*}$ \\
\hline \multicolumn{2}{|l|}{$\begin{array}{l}\text { Epidural haemorrhage by head } \\
\text { pin penetration }\end{array}$} & 1 \\
\hline \multirow{2}{*}{$\begin{array}{l}\text { Functional outcomes } \\
\mathrm{mRS}\end{array}$} & \multicolumn{2}{|c|}{ No of patients (in follow-up study) } \\
\hline & At discharge & After 3 years \\
\hline 0 & 62 & $62(59)$ \\
\hline 1 & 2 & $2(2)$ \\
\hline 2 & $1 \dagger$ & - \\
\hline 3 & $1 \neq$ & 19 \\
\hline 4 & - & - \\
\hline 5 & - & - \\
\hline 6 & $1 \S$ & 2 \\
\hline
\end{tabular}

mRS, modified Rankin Scale.

*One patient suffered seizure, minor infarction, and subdural hematoma. tCognitive function disorder lasting over 1 year after the operation. †This patient lost sight in the left eye on the morning of the 3rd perioperative day. §This patient suffered cardiopulmonary arrest on the 4th perioperative day - Cerebellar infarction occurred 2 years after the operation.

${ }^{* *}$ Massive putaminal haematoma occurred 1 year after treatment.

\section{Survey tools}

We assessed QOL using a Japanese version of Short Form-36 ( SF36), a self-administered QOL questionnaire that addresses eight domains (physical and social functioning, physical and emotional limitations, mental health, vitality, pain, and general health perception); these were presented as 36 topics and reflected subjective health perception. ${ }^{12-14}$ SF-36 data from a general Japanese population sample were used as reference scores. ${ }^{15}$ The Hospital Anxiety and Depression Scale (HADS) poses 14 questions developed to detect anxiety and depressive states; scores of $\leqslant 7$ are considered negative, scores of 8-10 as doubtful, and scores of $\geqslant 11$ recorded as positive. These scores do not solely reflect symptoms such as pain or motor weakness that may be experienced by individuals with physical illness. ${ }^{16}{ }^{17}$ The degree of disability was recorded using the scores of the modified Rankin Scale (mRS).${ }^{18} \mathrm{~A}$ recording of surgical complication was entered in cases manifesting neurological deficits or asymptomatic abnormal findings on CT or MRI studies within 30 days of the operation. Chronic subdural haematomas inside the craniotomy area were also regarded as surgical complications.

\section{Statistical analysis}

Each level of the eight domains on SF-36 was represented in terms of SD scores (norm-based score) calculated by the difference from the reference population. ${ }^{15}$ To assess statistical differences in the SF-36 domains and HADS scores between the study and reference populations, we analysed the difference between their mean scores using the Student's t test. For comparisons of values recorded before, and 3 months and 1 and 3 years after the operation, we analysed the mean SF-36 scores using the Wilcoxon matched-pairs test.

\section{RESULTS}

\section{Clinical characteristics and surgical results}

Of the 67 participating patients, 48 were women and 19 men; their mean age was 61.3 (10.4) years. They had a total of 78



Figure 1 Line chart showing the mean scores for the eight Short Form-36 (SF-36) domains in the operated patients and Japanese reference populations between 60 and 69 years of age.

aneurysms; 57 patients had 1, nine had two, and one had three aneurysms. Two patients with two and one patient with three aneurysms required two craniotomies. Of the 78 lesions, 27 were located at the internal carotid, 15 at the anterior cerebral, 31 at the middle cerebral and 5 at the vertebrobasilar arteries. The mean aneurysm size measured on three-dimensional CT scans or digital subtraction angiography scans was 5.2 (2.3) $\mathrm{mm}$ (range $2-10 \mathrm{~mm}$ ). The 78 aneurysms were treated in a total of 70 operations; in 73 aneurysms neck clipping was successful, the other 5 aneurysms were wrapped.

In $14(20.9 \%)$ patients we encountered neurological impairments and/or abnormal CT findings which were recorded as surgical complications. In most cases these complications were transient; five patients suffered transient neurological disorders such as epilepsy and dysphasia, two had asymptomatic abnormal findings on postoperative CT or MRI (putaminal infarction or contusional change), three had a chronic subdural haematoma requiring burr-hole evacuation, and two developed meningitis that was cured with antibiotics. One patient had a seizure with minor infarction and a chronic subdural haematoma; at discharge, this patient's condition had returned to normal. The postoperative follow-up survey included two patients with severe complications such as visual loss and cognitive impairment. We excluded six patients from the QOL follow-up survey; two experienced independent apoplexy, one died in the perioperative period of unknown causes and three failed to complete their survey forms. Thus, 61 patients were available for preoperative and postoperative study in this survey. As shown in table 1 , the mRS scores at 3 years after the operation were 0 in 59 patients and 1 in 2 patients.

\section{Health-related QOL before surgery}

Figure 1 depicts the preoperative mean scores for each of the 8 SF-36 domains in the 67 enrolled patients. Compared with the reference population, they had significantly lower scores $(\mathrm{p}<0.01)$ in the arena of social functioning, physical and emotional limitations, mental health, vitality and general health perception. With respect to physical functioning, the patients also manifested lower scores although the difference was not statistically significant. 


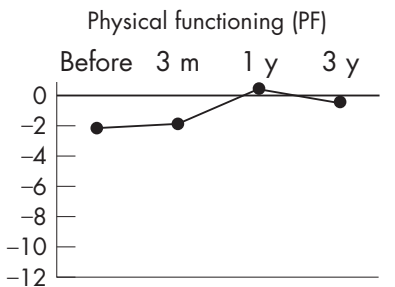

Emotional role limit (RE)



Bodily pain (BP)

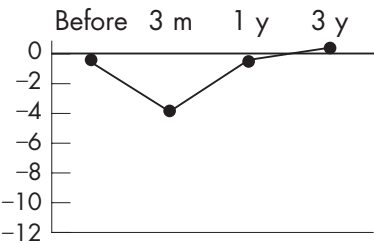

Social functioning (SF)

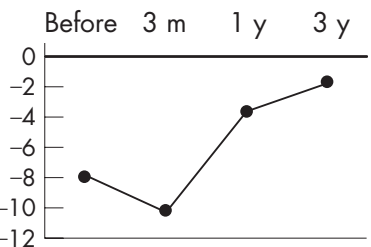

Mental health $(\mathrm{MH})$

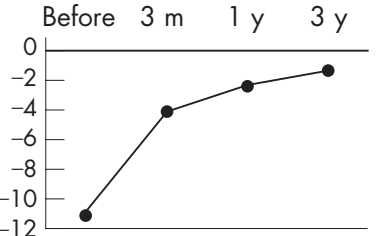

General health perception (GH)

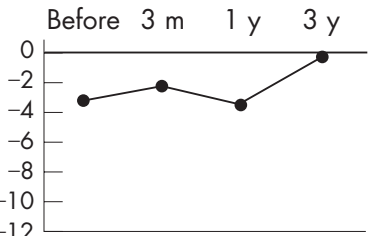

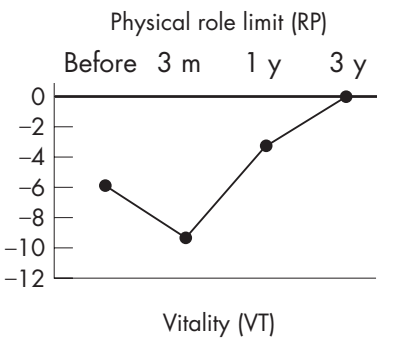

Figure 2 Change in each of the eight Short Form-36 (SF-36) domains before and $>3$ years after the operation. The scores of each SF-36 domain are shown as SD scores based on Japanese reference populations.

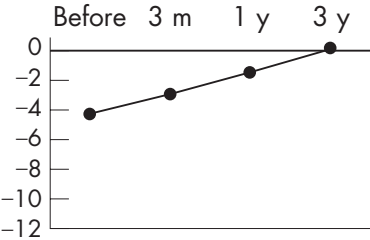

.

\section{Health-related QOL after surgery}

Of the 67 enrolled patients, 61 completed all pretreatment and post-treatment SF-36 questionnaires. Figure 2 represents changes in the health-related QOL over a 3-year postoperative period. Compared with their preoperative scores, scores at 3 months after the operation were significantly higher in the mental health domain $(p<0.01)$. Conversely, the bodily pain score was significantly lower $(p=0.04)$ and the scores for social functioning and for physical and emotional limitations were also lower. The scores for physical functioning before and 3 months after the operation were comparable. Compared with the preoperative scores, at 1 year after the operation, the mean SF-36 scores improved for physical and social functioning $(p=0.02)$, for physical $(p=0.05)$ and emotional limitations $(p=0.04)$, for mental health $(p<0.01)$ and for vitality $(p=0.04)$. On the other hand, compared with the reference scores, the patients' scores for social functioning and for physical and emotional limitations were significantly lower. At 3 years after the operation, all but the physical functioning scores were higher compared with those obtained at 3 months and 1 year after the operation. Moreover, for all domains listed

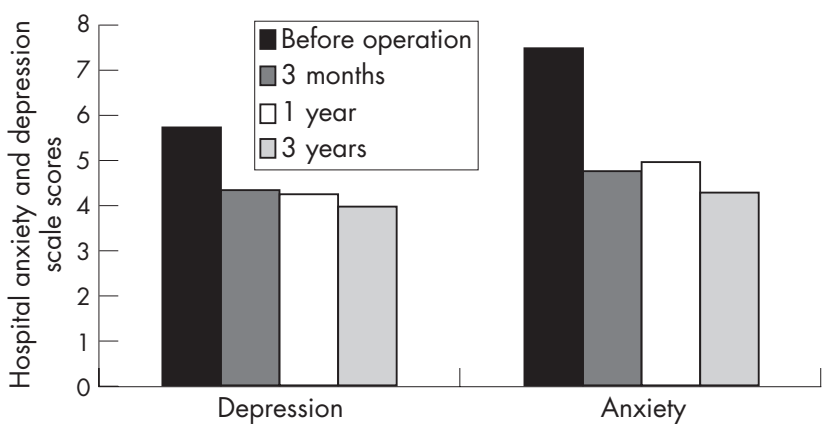

Figure 3 Preoperative and postoperative Hospital Anxiety and Depression Scale (HADS) for depression and anxiety. in the SF-36 questionnaire, at 3 years after the operation, the patient and reference scores were comparable.

\section{Changes in depression and anxiety}

The mean HADS subscores of the 62 patients for preoperative depression and anxiety were 5.7 (3.6) and 7.5 (4), respectively. These scores indicate that they did not experience serious clinical depression, and that their anxiety status was recorded as doubtful. Both subscores decreased after surgical treatment. There was a statistically significant difference between the patients' preoperative and postoperative depression and anxiety scores (fig 3).

\section{DISCUSSION}

To our knowledge, this is the first report monitoring the longterm self-assessed QOL of patients who underwent elective surgery for incidentally found unruptured ICAs. Using SF-36 and HADS, we found that before the operation, the 67 patients initially enrolled in this survey presented with a markedly lower QOL than the Japanese reference population. We observed a transient decline to below the preoperative score in some of the eight SF-36 domains at 3 months after the operation. However, at 3 years postoperatively, the QOL scores of the 61 operated patients who completed the survey had returned to the mean QOL scores of the reference population.

According to the MARS study group, ${ }^{19}$ some of their 17 patients who underwent elective surgery for unruptured ICAs showed improvement in their QOL; however, none had returned to their preoperative levels at l year after the operation. Although many patients experience a QOL decline within the first postoperative year, our study showed that after 3 years, the scores of the patient population and the reference population were comparable for all domains listed in the SF-36 questionnaire. This suggests that the appropriateness of elective surgery for unruptured ICAs should be considered in terms of preventing SAH and also from the perspective of an improved QOL.

In our survey, psychosocial activities were evaluated by scoring social functioning and physical and emotional limita- 
tions on SF-36. The increase in scores rating these domains was markedly delayed, whereas the score for mental health, rated by HADS, recovered within 3 months of the operation. At 3 months postoperatively, the presence of persistent headache, indicated by an increase in the bodily pain score, may reduce the patients' psychosocial activities. Betchen et al ${ }^{10}$ reported a similar short-term QOL decrease in patients operated for acoustic neuromas. In our study, the scores for social functioning and physical and emotional limitations had not recovered completely as late as 1 year after the operation, when cessation of treatment-related headaches can be expected. Since endovascular treatment did not affect the QOL of patients negatively, the low psychosocial activity scores of operated patients may reflect the effect of invasive surgical procedures such as retraction of the frontal lobe, or compromised venous circulation. ${ }^{19} 20$ According to van der Schaff et al, ${ }^{21}$ patients aware of the presence of untreated ICAs continued to manifest a decrease in psychosocial function for over 3.75 years. Thus, our results that the patients' QOL had fully recovered 3 years after treatment can be interpreted to argue for surgical treatment.

Like others, ${ }^{19}$ we found that operative complications may not have long-term negative effects on the postoperative QOL. Among 14 (20.9\%) patients with surgical complications according to our stringent criteria, two manifested severe neurological impairments. One of those, a 54-year-old woman with an unruptured aneurysm at the left internal carotid artery, developed ipsilateral visual disturbance after the operation; however, her QOL recovered and her mRS changed from grade 3 to grade 1 in the course of 3 years. The other patient, a 76year-old woman with post-treatment mental disorder, experienced a good post-treatment QOL and improvement in her daily activities; her mRS grade was 0 at 3 years. Our previously reported retrospective study ${ }^{11}$ of electively operated patients with unruptured ICAs also showed that a history of cardiac disease did affect the postoperative QOL whereas operative complications did not. As facial paresis did not affect the selfassessed QOL in patients operated for acoustic neuromas, ${ }^{10}$ the possibility of a discrepancy between objectively judged neurological impairments and the self-assessed QOL cannot be ruled out.

Long-term results with respect to the prevention of SAH and improved QOL scores in patients treated endovascularly for unruptured aneurysms remain to be determined. Patients treated by coil embolisation must undergo periodic angiographic study and may require additional intervention which may affect their QOL negatively. ${ }^{20}$ At present, there is no optimal strategy for dealing with unruptured ICAs. Therefore, the physician and patient must carefully consider QOL issues in reaching treatment decisions. In their cost-effectiveness study, King $e t \mathrm{al}^{22}$ proposed that a QOL decrease in patients harbouring aneurysms represents an indication for elective surgery. We continue to recommend intervention in patients at risk for aneurysmal rupture. In patients with asymptomatic ICAs or meningiomas, the postoperative QOL is an important outcome measure because the postoperative prognosis of these patients tends to be good. This information may guide the patient's decision on whether to undergo elective surgery.

In conclusion, our study showed that although the QOL of patients who underwent elective surgery for unruptured ICAs declined in the short term, it recovered in the course of 3 years. Treatment decisions must consider surgical risks and the prevention of SAH, and also the possible improvement of the patients' QOL.

\section{Authors' affiliations}

Shigeo Yamashiro, Kumamoto Rosai Hospital, Kumamoto, Japan Toru Nishi, Kazunari Koga, Tomoaki Goto, Masatomo Kaji, Daisuke Muta, Shodo Fujioka, Department of Neurosurgery, Stroke Centre, Saiseikai Kumamoto Hospital, Kumamoto, Japan

Jun-ichi Kuratsu, Department of Neurosurgery, Kumamoto University

Graduate School of Medicine, Kumamoto, Japan

Competing interests: None declared.

Ethical approval: This study was approved by the ethics committee of the Saiseikai Kumamoto Hospital.

\section{REFERENCES}

1 Bederson JB, Awad IA, Wiebers DO, et al. Recommendations for the management of patients with unruptured intracranial aneurysms: a statement for health care professionals from the Stroke Council of the American Heart Association. Stroke 2000;31:2742-50.

2 Morita A, Fujiwara S, Hashi K, et al. Risk of rupture associated with intact cerebral aneurysms in the Japanese population: a systematic review of the literature from Japan. J Neurosurg 2005;102:601-6.

3 The International Study of Unruptured Intracranial Aneurysms Investigators. Unruptured intracranial aneurysms-risk of rupture and risks of surgical intervention. N Engl J Med 1998;339:1725-33.

4 Wiebers DO, Whisnant JP, Huston J 3rd, et al. International study of unruptured intracranial aneurysms investigators. Unruptured intracranial aneurysms: natura history, clinical outcome, and risks of surgical and endovascular treatment, Lancet 2003;362:103-10.

5 Raaymakers TWM, Rinkel GJ, Limburg M, et al. Mortality and morbidity of surgery for unruptured intracranial aneurysms: a meta-analysis. Stroke 1998;29:1531-8.

6 Deane M, Pigott T, Dearing P. The value of the Short Form 36 score in the outcome assessment of subarachnoid haemorrhage. $\mathrm{Br} J$ Neurosurg 1996;10:187-91.

7 Hop JW, Rinkel GJE, Algra A, et al. Quality of life in patients and partners after aneurysmal subarachnoid hemorrhage. Stroke 1998:29:798-804.

8 Hütter BO, Kreitschmann-Andermahr I, Gilsbach JM. Health-related quality of life after aneurysmal subarachnoid hemorrhage: impacts of bleeding severity, computerized tomography findings, surgery, vasospasm, and neurological grade. J Neurosurg 2001;94:241-51.

9 Czechowsky D, Hill MD. Neurological outcome and quality of life after stroke due to vertebral artery dissection. Cerebrovasc Dis 2002;13:192-97.

10 Betchen SA, Walsh J, Post KD. Self-assessed quality of life after acoustic neuroma surgery. J Neurosurg 2003;99:818-23.

11 Yamashiro S, Nishi T, Koga K, et al. Validation of surgical treatment for asymptomatic unruptured intracranial aneurysms in view of patient quality of life. Surg Cerebral Stroke. 2005;33: 8-13 (in Japanese))

12 Ware JE Jr, Sherbourne CD. The MOS 36-item short-form health survey (SF-36) I. Conceptual framework and item selection. Med Care 1992;30:473-83.

13 Fukuhara S, Bito S, Green J, et al. Translation, adaptation, and validation of the SF-36 Health Survey for use in Japan. J Clin Epidemiol 1998;51:1037-44.

14 Fukuhara S, Ware JE Jr, Kosinski M, et al. Psychometric and clinical tests of validity of the Japanese SF-36 Health Survey. J Clin Epidemiol 1998:51:1045-53.

15 Fukuhara S, Suzukamo Y, Bito S, et al. Manual of SF-36 Japanese version 1.2. Tokyo, Japan: Public Health Research Foundation, 2001.

16 Zigmond AS, Snaith RP. The hospital anxiety and depression scale. Acto Psychiatr Scand 1983;67:361-70.

17 Zigmond AS, Snaith RP, Kitamura T. Hospital anxiety and depression scale. Seishinka Shindangaku. 1993;4: 371-2 (in Japanese)l.

18 Bonita R, Beaglehole R. Recovery of motor function after stroke. Stroke 1988; 19:1497-1500.

19 Raaymakers TWM, MARS Study Group. Functional outcome and quality of life after angiography and operation for unruptured intracranial aneurysms. J Neuro Neurosurg Psychiatry 2000;68:571-6.

20 Brilstra EH, Rinkel GJE, van der Graaf Y, et al. Quality of life after treatment of unruptured intracranial aneurysms by neurosurgical clipping or by embolisation with coils. Cerebrovasc Dis 2004:17:44-52.

21 van der Schaff IC, Brilstra EH, Rinkel GJE, et al. Quality of life, anxiety, and depression in patients with an unruptured intracranial aneurysm or arteriovenous malformation. Stroke 2002;33:440-3.

22 King JT, Glick HA, Mason TJ, et al. Elective surgery for asymptomatic, unruptured, intracranial aneurysms: a cost-effectiveness analysis. J Neurosurg 1995;83:403-12. 